\title{
Análise da qualidade da informação da mortalidade prematura por doenças crônicas não transmissíveis e sua utilização nos Objetivos de Desenvolvimento Sustentável
}

\section{Analysis of the quality of information on premature mortality due to chronic noncommunicable diseases and its use in the Sustainable Development Goals}

\author{
Jéssica Muzy¹ (D), Débora Castanheira' (D), Dalia Romero' (D) \\ 'Grupo de Informação em Saúde e Envelhecimento, Instituto de Comunicação e Informação Científica e Tecnológica (ICICT), \\ Escola Nacional de Saúde Pública (ENSP), Fundação Oswaldo Cruz (FIOCRUZ) - Rio de Janeiro (RJ), Brasil.
}

Como citar: Muzy J, Castanheira D, Romero D. Análise da qualidade da informação da mortalidade prematura por doenças crônicas não transmissíveis e sua utilização nos Objetivos de Desenvolvimento Sustentável. Cad Saúde Colet, 2021;29(esp.):152-164. https://doi.org/10.1590/1414-462X202199010456

\section{Resumo}

Introdução: A taxa de mortalidade prematura (TMP) por doenças crônicas não transmissíveis (DCNT) é um indicador utilizado pela Organização das Nações Unidas (ONU) para acompanhar uma das metas propostas nos Objetivos de Desenvolvimento Sustentável (ODS). Ela permite medir a eficácia e a efetividade das ações de prevenção, tratamento e promoção da saúde desde que seja estimada com informações confiáveis e de qualidade. Objetivo: Este estudo tem como objetivo analisar as potencialidades, as limitações e a completude das informações utilizadas para o cálculo e a análise desse indicador. Método: Foram utilizadas as informações de óbitos do Sistema de Informações Sobre obre Mortalidade (SIM) de 2000 a 2016. Foi estimada a proporção de óbitos por causas mal definidas, bem como o percentual de incompletude das variáveis raça/cor, escolaridade, ocupação, estado civil, naturalidade e assistência médica. Resultados: Cerca de 68\% dos municípios apresentaram qualidade adequada da causa de óbito. A raça/cor foi a única que, em geral, já tinha boa completude. O SIM se mostrou uma boa fonte de dados para estimar a TMP. Apesar disso, sua qualidade não é a mesma em todas as abrangências. Conclusão: Embora a maioria dos municípios apresente boa completude da informação, muitos ainda apresentam péssimo preenchimento, sendo necessário identificá-los para não estimar um indicador pouco confiável. Palavras-chave: mortalidade prematura; doenças crônicas; sistemas de informação.

\section{ABSTRACT}

Background: The premature mortality rate (PMR) from noncommunicable chronic diseases (NCD) is an indicator used by the United Nations (UN) to track one of the objectives proposed in the Sustainable Development Goals (SDG). The PMR measures the effectiveness of prevention, treatment and health promotion actions, but the sources used to estimate this indicator must be reliable. Objective: This study aims to analyze the potentialities, limitations, and completeness of the information used to calculate and analyze this indicator. Method: We used information on mortality from the Mortality Information System (SIM) from 2000 to 2016. The proportion of deaths with poorly defined causes and the percentage of

\footnotetext{
Trabalho realizado no Instituto de Comunicação e Informação Científica e Tecnológica em Saúde (ICICT), da Fundação Oswaldo Cruz (Fiocruz), Rio de Janeiro (RJ), Brasil.

Correspondência: Jéssica Muzy Rodrigues. E-mail: jehmuzy@gmail.com

Fonte de financiamento: nenhuma.

Conflito de interesses: nada a declarar.

Recebido em: Set. 22, 2019. Aprovado em: Dez. 26, 2019
}

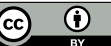

Este é um artigo publicado em acesso aberto (Open Access) sob a licença Creative Commons Attribution, que permite uso, distribuição e reprodução em qualquer meio, sem restrições desde que o trabalho original seja corretamente citado. 
incompleteness of the variables race, level of education, occupation, marital status, place of birth, and medical care were estimated. Results: Approximately $68 \%$ of the municipalities presented adequate quality of information on the cause of death. Race was the only variable that presented good overall completeness. Conclusion: The SIM has proved to be a good source of data for estimating the PMR; however, its quality is not the same across ranges. Although most municipalities present good data completeness, many are still poorly filled, and it is necessary to identify them so that a reliable indicator can be estimated.

Keywords: mortality; premature; chronic disease; information systems.

\section{INTRODUÇÃO}

O perfil de agravos que mais afetam a população passou das doenças infectocontagiosas para as crônicas não transmissíveis, fenômeno conhecido como transição epidemiológica'. Em 2016, último ano em que foram disponibilizadas as informações de mortalidade, o grupo das principais doenças crônicas não transmissíveis (DCNT) já representava $58,8 \%$ das causas de morte no Brasil na população geral e $56 \%$ na faixa etária de 30 a 69 anos. A necessidade de estabelecer medidas para o enfrentamento das doenças crônicas foi reconhecida nacional e internacionalmente por meio de políticas, programas e acordos internacionais.

Em 2015, a Organização das Nações Unidas (ONU) publicou documento, do qual o Brasil é signatário, definindo 17 Objetivos de Desenvolvimento Sustentável (ODS)². Cada um desses objetivos tem um conjunto de metas a serem alcançadas e, como medida de desempenho, indicadores designados.

O objetivo 3 dos ODS busca estimular a saúde de qualidade, assegurando uma vida saudável e promovendo o bem-estar para todos, em todas as idades. Na meta 3.4 desse objetivo, é apontado o uso da taxa de mortalidade prematura (TMP) como uma medida da eficácia e efetividade das ações de prevenção, tratamento e promoção da saúde. A meta proposta é de que a TMP seja reduzida em um terço até 2030 . O indicador pode ser utilizado tanto para acompanhar o processo quanto para avaliar as intervenções realizadas.

É importante notar que o uso desse indicador não é novo no Brasil. O Plano de Ações Estratégicas para o Enfrentamento das Doenças Crônicas Não Transmissíveis, de 2011³, também identifica pontos de intervenção para combate dessas doenças:

[...] as desigualdades sociais, as diferenças no acesso aos bens e aos serviços, a baixa escolaridade, as desigualdades no acesso à informação, além dos fatores de risco modificáveis, como tabagismo, consumo de bebida alcoólica, inatividade física e alimentação inadequada, tornando possível sua prevenção ${ }^{3: 8}$.

O Plano também aponta o uso da TMP para o monitoramento de tendências e avaliação das melhorias realizadas na implementação de estratégias e planos sobre doenças não transmissíveis. A meta para o Brasil é reduzir $2 \%$ ao ano. Além disso, o Plano aponta uma série de indicadores para controle dos fatores de risco relacionados às DCNT. Quanto às desigualdades socioeconômicas na mortalidade prematura, embora reconhecidas, não são monitoradas.

Tanto no tocante aos ODS quanto ao Plano, é importante notar que, para que essas metas e objetivos propostos sejam devidamente monitorados, é fundamental dispor de informações oportunas e adequadas que permitam realizar esse acompanhamento das políticas ${ }^{4}$. A informação em saúde é central para conhecer a situação de saúde da população e realizar o acompanhamento de políticas e programas ${ }^{5}$. No entanto, para assegurar que as informações utilizadas sejam confiáveis, é necessário analisar a sua qualidade, considerando alguns critérios, entre os quais:

1. A clareza metodológica quanto às instruções de coleta, manuais de preenchimento e documentação da base de dados é fundamental para permitir a comparabilidade das variáveis com outras fontes de informação no que se refere a conceitos e definições.

2. A acessibilidade, levando em conta a forma de disponibilização dos dados (valor e meio físico ou eletrônico), o tipo de informação disponível (individual ou agregada), o local e o 
procedimento de pedido dos dados, o tempo de entrega após o pedido ao responsável e os formatos dos arquivos ${ }^{6}$.

3. A oportunidade do sistema, que se refere ao tempo necessário até que as informações estejam disponíveis para acesso pelo usuário.

4. A completude/incompletude, que diz respeito ao grau de preenchimento dos campos do instrumento de coleta, nesse caso, a declaração de óbito.

A não conformidade a esses critérios, principalmente no que tange à completude das informações, diminuiu a utilidade do indicador no monitoramento e avaliação de políticas públicas ${ }^{6}$.

Não existem estudos no Brasil que comprovem que os Sistemas de Informação em Saúde têm informação de qualidade para calcular o indicador de TMP. Tendo isso em vista, o objetivo deste artigo é analisar a qualidade das informações epidemiológicas, demográficas e socioeconômicas utilizadas para o cálculo e uso da TMP por DCNT disponíveis no Brasil e, dessa forma, determinar sua aplicabilidade para o monitoramento dos ODS no país.

\section{MATERIAIS E MÉTODOS}

A principal fonte de informação utilizada neste estudo é o Sistema de Informações Sobre Mortalidade (SIM). Os microdados de mortalidade foram obtidos a partir do Departamento de Informática do SUS (DATASUS) do Ministério da Saúde. Para este estudo, foram selecionados os óbitos da população na faixa etária de 30 a 69 anos do Brasil por regiões, unidades da federação (UF) e municípios, entre 2000 e 2016, último ano disponível no momento da elaboração deste artigo. Algumas análises foram feitas por faixa etária, considerando a concentração de óbitos prematuros na faixa de 60 a 69 anos de idade.

A definição de mortalidade prematura utilizada neste estudo é a mesma da OMS². São consideradas mortes prematuras os óbitos na faixa de 30 a 69 anos causados pelo conjunto das principais DCNT, sendo elas: as doenças do aparelho circulatório, câncer, diabetes mellitus e doenças respiratórias crônicas (CID-10: I00-199; C00-C97; J30-J98; E10-E14)7. As estimativas calculadas para este estudo foram realizadas utilizando o Statistical Package for the Social Science (SPSS), versão 21.

A clareza metodológica refere-se a instruções de coleta, manuais de preenchimento e documentação da base de dados, assim como à comparabilidade das variáveis com outras fontes de informação quanto aos conceitos e definições, e foi avaliada considerando a precisão dos conceitos e definições, além da concordância entre os diferentes documentos metodológicos do SIM.

Para analisar a acessibilidade, foram avaliados a sua disponibilidade (preço e forma de aquisição - meio físico ou eletrônico), o tipo de informação disponível (individual ou agregada), o local e o procedimento de pedido dos dados, o tempo de entrega após o pedido ao responsável e os formatos dos arquivos.

A oportunidade dos dados foi analisada levando em consideração o tempo entre a entrega dos resultados e o período de referência estabelecido para a disponibilidade dos dados ao usuário.

Para analisar a completude/incompletude, foi utilizada a 10a revisão da Classificação Internacional de Doenças (CID10) ${ }^{7}$ em níveis de três caracteres dos códigos da causa básica de morte. As análises foram realizadas de acordo com os seguintes níveis de agregação: regional, estadual, municipal e individual.

\section{Proporção de óbitos por causas mal definidas e classificação da qualidade de informação}

A causa básica de óbito é uma variável fundamental para estimar a TMP, pois a partir dela é possível identificar a "doença ou lesão que iniciou a cadeia de acontecimentos patológicos que conduziram diretamente à morte, ou as circunstâncias do acidente ou violência que produziram a lesão fatal"10 (p. 26). 
Para analisar a completude/incompletude das informações sobre mortalidade, foi utilizado o indicador proporção de óbitos por causas mal definidas (PCMD). As causas mal definidas de óbito pertencem ao capítulo XVIII da CID10. Nesse capítulo, são considerados os sintomas, sinais e achados anormais de exames clínicos e de laboratório não classificados em outra parte, que correspondem aos códigos R00 a R99. Seu cálculo é representado pela seguinte Fórmula 1:

PCMD $=(($ óbitos do capitulo XVIII $) /($ total de óbitos $)) * 100$

Para a análise da evolução temporal da qualidade das informações de mortalidade, foi calculada a PCMD para o período entre 2000 e 2015. A tendência no período foi analisada segundo as faixas etárias (30-59 anos e 60-69 anos) por regiões geográficas.

A PCMD também foi utilizada para estimar a qualidade das informações sobre a mortalidade na faixa etária de 30 a 69 anos e quantificar os municípios segundo uma classificação de qualidade de registro. Essa classificação foi feita com base em um banco agregado por municípios, no qual foram excluídos os municípios ignorados, que são aqueles que pertencem a alguma UF, mas não é possível identificá-los a partir dos códigos registrados.

Foi calculada a PCMD para cada um dos municípios, e, em seguida, estes foram classificados quanto à qualidade da informação. A classificação da qualidade tomou como referência valores estabelecidos na literatura ${ }^{8}$ para escalonar os níveis de adequação da informação, sendo eles: PCMD menor do que $5 \%(<5 \%)$ : qualidade altamente adequada.

PCMD entre $5 \%$ e $7 \%$ (= 5\% e < $7 \%$ ): qualidade adequada.

PCMD entre $7 \%$ e $10 \%$ (= $7 \%$ e $<10 \%)$ : qualidade pouco adequada.

PCMD entre $10 \%$ e $15 \%$ (= $10 \%$ e < 15\%): qualidade inadequada.

PCMD igual ou maior do que 15\% (= ou > 15\%): qualidade altamente inadequada.

\section{Completude das variáveis socioeconômicas}

Como uma dimensão da qualidade das informações, é considerada também a completude das variáveis socioeconômicas do SIM, que coleta, em nove blocos, aproximadamente 40 variáveis por meio das Declarações de Óbitos (DO). As informações socioeconômicas consideradas para estudo da desigualdade na mortalidade de idosos correspondem às seguintes variáveis socioeconômicas da DO: naturalidade, idade, sexo, raça ou cor da pele, situação conjugal, escolaridade e ocupação.

Para analisar a sua completude, foi estimada para o Brasil, regiões e municípios a proporção de campos em branco (não preenchidos) e de informação classificada como ignorada (especificada no manual de preenchimento da DO com o código 9) ou códigos inexistentes. A inclusão desse código foi adotada diante das evidências de que a informação classificada como "Ignorada" corresponde, em sua maioria, a campos sem informação, e não a desconhecimento do informante ${ }^{9}$. O mau preenchimento da DO também foi objeto de análise deste trabalho.

O indicador de completude/incompletude (Fórmula 2) das variáveis socioeconômicas consiste no número de $\mathrm{DO}$ de idosos com mau preenchimento da variável socioeconômica selecionada, dividido pelo total de óbitos e multiplicado por 100.

Incompletude $=(($ número óbitos com variável X mal preenchida $) /($ total de óbitos $)) * 100$

Para identificar os municípios quanto à qualidade do preenchimento do conjunto das variáveis socioeconômicas selecionadas, foi utilizada a classificação de acordo com os seguintes graus de avaliação da incompletude:

"Excelente": $<5 \%$.

"Bom": $5 \%$ a 9,99\%.

"Regular": $10 \%$ a 19,99\%.

"Ruim": 20\% a 49,99\%.

"Muito ruim": > 50\%. 


\section{RESULTADO}

Em relação à acessibilidade, as informações do SIM são disponibilizadas gratuitamente por meio de plataforma on-line e em âmbito individual. Os dados podem ser tabulados na própria página ou baixados para utilização em TABWIN (software desenvolvido pelo Ministério da Saúde para tabulação off-line dos dados do SIM) ou outros softwares. Algumas informações sobre o local de residência do falecido são omitidas do sistema para garantir a privacidade, no entanto essa é uma recomendação internacional para segurança. Ainda assim, a omissão desse tipo de informação limita a realização de estudos mais específicos sobre a desigualdade espacial em saúde.

Alguns problemas podem ser observados quanto à oportunidade dos dados do SIM. Apesar de o acesso aos dados ser imediato na busca por meio eletrônico, no momento da finalização deste artigo (maio 2019), existia uma defasagem de mais de dois anos entre a ocorrência do óbito e a disponibilidade dos dados.

Quanto à completude/incompletude, que se refere aos campos em branco e aos códigos atribuídos à informação ignorada especificada no manual de preenchimento da DO, foi verificado que o mau preenchimento das variáveis geralmente está associado ao uso de expressões ou termos imprecisos. Dependendo da magnitude da incompletude na variável selecionada, é perdido um percentual muito elevado de dados que prejudicam ou inviabilizam realizar aferições a partir dessa informação. O mau preenchimento dessa variável causa a perda de informação epidemiológica fundamental, o que pode levar à estimação de um indicador impreciso e pouco confiável.

O indicador utilizado para analisar a completude do preenchimento da causa básica de óbito foi a PCMD. Esse indicador é amplamente utilizado na literatura para avaliar a qualidade da informação de mortalidade ${ }^{11}$. A Tabela 1 aponta quantos municípios brasileiros possuíam, em 2016, adequada informação da causa básica de óbito. Foi verificado que, dos 5.393 municípios com casos de óbitos para o ano de $2016,68,8 \%$ tinham informações altamente adequadas ou adequadas quanto à causa básica de óbito.

Tabela 1. Classificação dos municípios quanto à proporção de óbitos por causas mal definidas (PCMD) na população de 30 a 69 anos, Brasil, 2016

\begin{tabular}{ccccc}
\hline Classificação & $\mathbf{n}$ & \% válida & \% acumulativa \\
\hline $\begin{array}{c}\text { Altamente } \\
\text { adequado }\end{array}$ & $(<5 \%)$ & 2986 & 55,4 & 55,4 \\
\hline Adequado & $(5 \%-<7 \%)$ & 725 & 13,4 & 68,8 \\
\hline Pouco adequado & $(7 \%-<10 \%)$ & 647 & 12 & 80,8 \\
\hline Inadequado & $(10 \%-<15 \%)$ & 588 & 10,9 & 91,7 \\
\hline $\begin{array}{c}\text { Altamente } \\
\text { inadequado }\end{array}$ & $(>=15 \%)$ & 447 & 8,3 & 100 \\
\hline & Total & 5393 & 100 & -
\end{tabular}

Fonte: Sistema de Informações Sobre Mortalidade (SIM) ${ }^{25}$

A faixa etária de 60 a 69 anos de idade apresentou maior redução da PCMD em relação à de 30 a 59 anos. No entanto, na análise por regiões, a PCMD não apresentou tantas variações quanto para municípios. As regiões Nordeste e Norte foram as que apresentaram maior redução de registros de morte por causas mal definidas, como pode ser observado na Figura 1. Foi constatada, nos anos de 2005 e 2007, uma queda brusca nos óbitos por causas mal definidas nessas regiões. 
$30-59$ anos

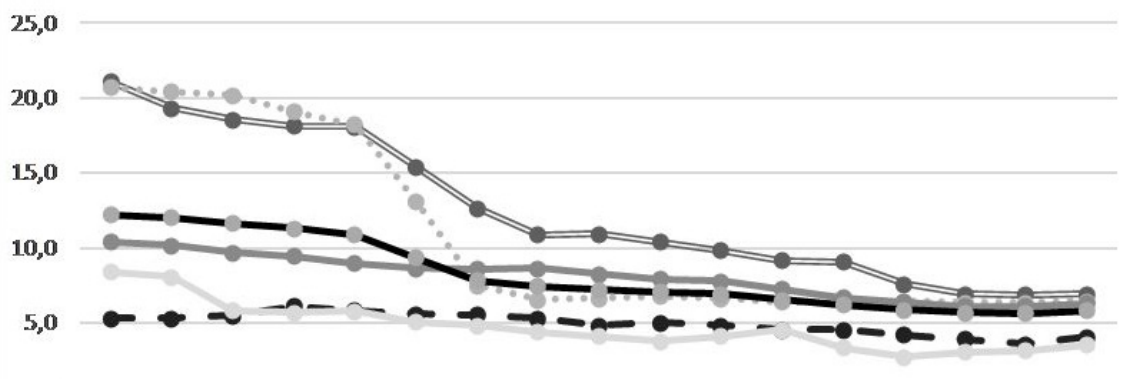

0,0

20002001200220032004200520062007200820092010201120122013201420152016

$\Longrightarrow$ NO $\cdots$. NE $\longrightarrow$ SE $\longrightarrow$ -

$60-69$ anos

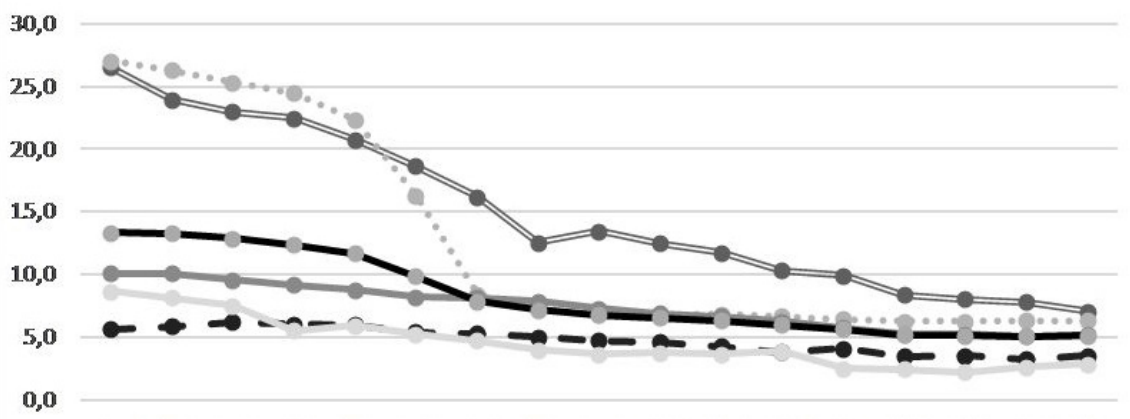

20002001200220032004200520062007200820092010201120122013201420152016

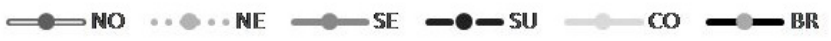

Total

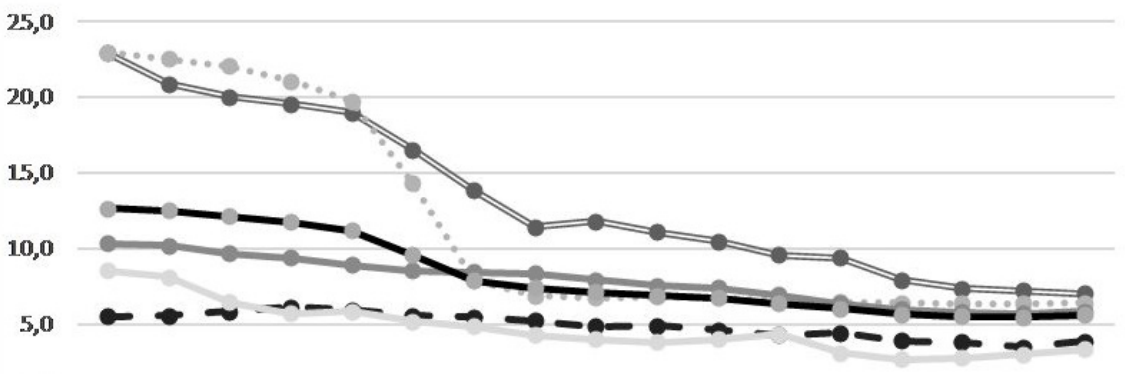

0,0

20002001200220032004200520062007200820092010201120122013201420152016

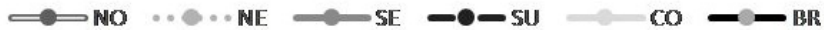

Figura 1. Proporção de óbitos por causas mal definida (PCMD), segundo regiões e faixa etária. Brasil, 20002016. Fonte: Sistema de Informações Sobre Mortalidade (SIM)

Na Tabela 2 verificou-se que a PCMD, inicialmente mais elevada entre os idosos (6069 anos), não apresentou diferenças significativas segundo o grupo etário nos anos mais recentes. Contudo, é importante notar que, apesar da notável melhora no preenchimento da variável causa básica de óbito, em 2016 apenas as regiões Sul e Centro-Oeste alcançaram preenchimento altamente adequado. 


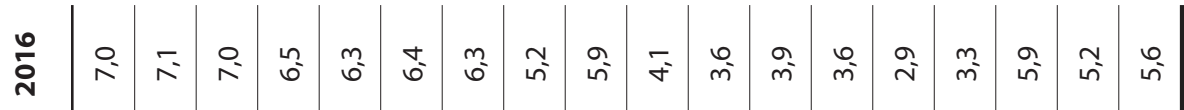

商

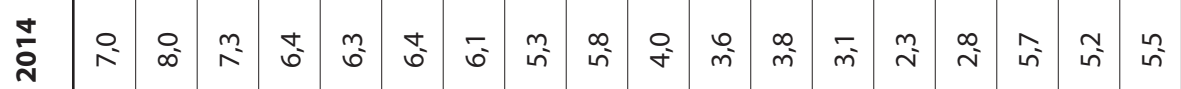

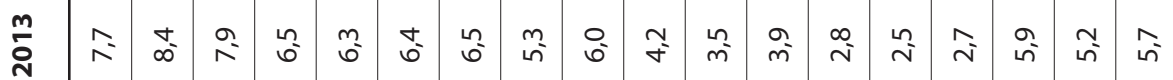

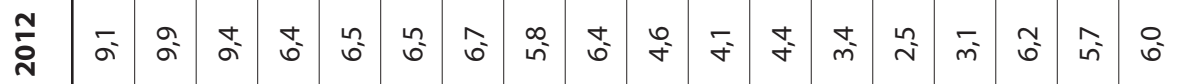

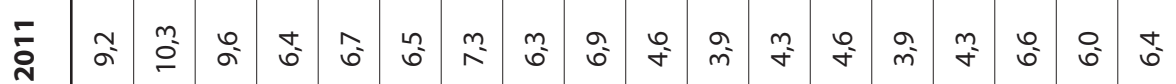

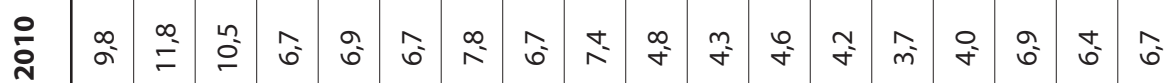

Oे

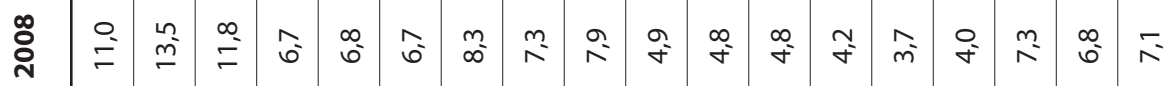

ồे

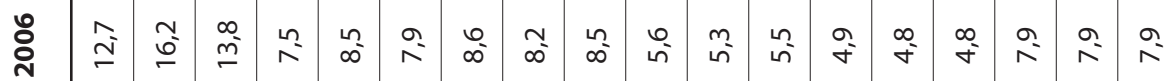

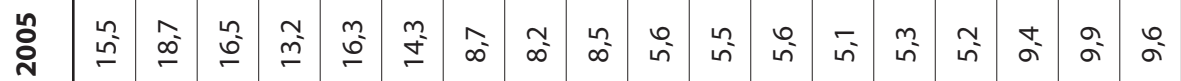

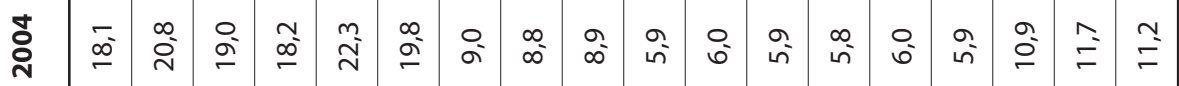

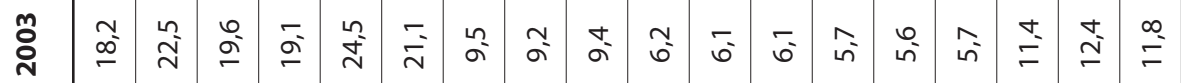

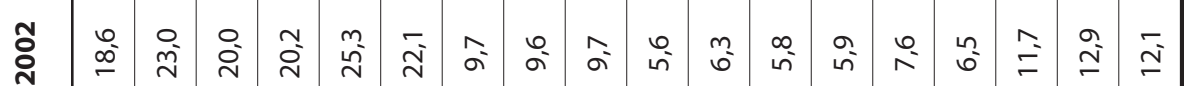

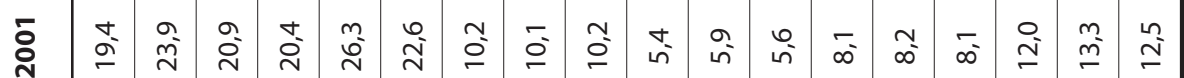

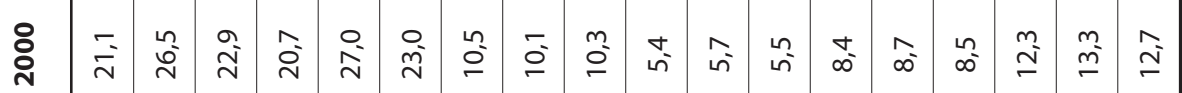

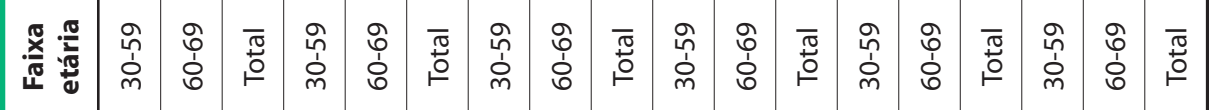

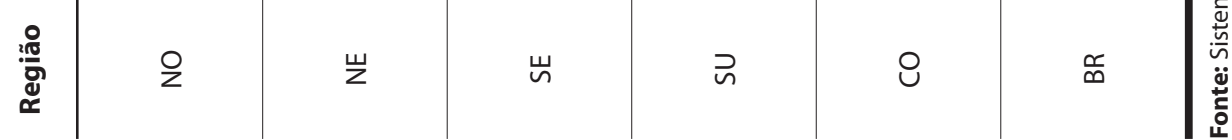


Outro aspecto relevante para a análise da qualidade da informação relacionada a mortes prematuras por DCNT é a completude/incompletude das informações socioeconômicas e demográficas disponíveis no SIM. Com a análise desses critérios, é esperado também evidenciar o potencial e as limitações dessas informações para a produção de estudos sobre a desigualdade na mortalidade segundo as causas específicas.

Na Tabela 3, é encontrado o indicador de completude por estado. É possível observar que, para o ano de 2016, a variável que apresentou melhor qualidade da informação foi raça/ cor. Quase todos os estados tiveram a incompletude abaixo de $10 \%$, classificados então com preenchimento excelente ou bom. Houve algumas notáveis exceções, como Alagoas (13,8\%) e Espírito Santo (12,4\%), ambos considerados regulares.

Tabela 3. Incompletude das variáveis socioeconômicas e demográficas do Sistema de Informações Sobre Mortalidade (SIM) segundo as unidades da federação (UF) de ocorrência do óbito. Brasil, 2016

\begin{tabular}{|c|c|c|c|c|c|c|}
\hline Região/UF & Raça/cor & Escolaridade & Ocupação & Estado civil & Naturalidade & $\begin{array}{l}\text { Assistência } \\
\text { médica }\end{array}$ \\
\hline NO & 2,1 & 18,7 & 24,9 & 14,1 & 8,6 & 29,1 \\
\hline Rondônia & 1,5 & 20,1 & 21,9 & 13,7 & 17,2 & 21,6 \\
\hline Acre & 3,1 & 23,7 & 40,8 & 22,4 & 5,8 & 58,3 \\
\hline Amazonas & 1,9 & 17,1 & 19,5 & 13,4 & 3,8 & 32,9 \\
\hline Roraima & 2,2 & 19,7 & 21,9 & 20,2 & 2,0 & 58,6 \\
\hline Pará & 1,8 & 17,7 & 26,1 & 13,3 & 10,9 & 26,1 \\
\hline Amapá & 3,0 & 22,2 & 41,0 & 17,3 & 5,7 & 34,8 \\
\hline Tocantins & 3,5 & 21,3 & 20,4 & 12,8 & 2,4 & 18,3 \\
\hline NE & 6,0 & 25,0 & 25,2 & 14,0 & 5,7 & 36,0 \\
\hline Maranhão & 2,6 & 13,3 & 21,8 & 10,4 & 8,1 & 32,0 \\
\hline Piauí & 5,9 & 17,9 & 19,3 & 13,6 & 6,9 & 20,0 \\
\hline Ceará & 6,0 & 15,3 & 22,6 & 8,1 & 4,9 & 25,8 \\
\hline Rio Grande do Norte & 7,1 & 28,8 & 37,5 & 12,6 & 4,1 & 48,2 \\
\hline Paraíba & 6,0 & 52,7 & 44,7 & 22,8 & 2,9 & 52,8 \\
\hline Pernambuco & 2,0 & 15,7 & 14,6 & 7,8 & 5,5 & 25,3 \\
\hline Alagoas & 13,8 & 51,7 & 43,8 & 31,6 & 20,3 & 38,6 \\
\hline Sergipe & 3,0 & 15,7 & 18,6 & 10,0 & 7,5 & 29,7 \\
\hline Bahia & 8,7 & 29,4 & 24,6 & 17,9 & 2,8 & 47,4 \\
\hline SE & 3,7 & 23,7 & 21,0 & 9,0 & 11,3 & 44,4 \\
\hline Minas Gerais & 7,7 & 32,8 & 28,6 & 14,6 & 6,7 & 43,3 \\
\hline Espírito Santo & 12,4 & 46,1 & 21,6 & 19,1 & 16,2 & 27,3 \\
\hline Rio de Janeiro & 1,3 & 13,8 & 21,5 & 5,3 & 29,8 & 54,8 \\
\hline São Paulo & 2,3 & 22,6 & 17,3 & 7,4 & 4,2 & 41,3 \\
\hline SU & 2,8 & 18,5 & 13,6 & 8,3 & 4,7 & 21,3 \\
\hline Paraná & 1,8 & 8,7 & 8,5 & 5,4 & 2,8 & 12,8 \\
\hline Santa Catarina & 1,4 & 12,4 & 12,1 & 8,1 & 5,0 & 23,8 \\
\hline Rio Grande do Sul & 4,3 & 29,6 & 18,7 & 10,9 & 6,3 & 27,3 \\
\hline CO & 2,5 & 20,4 & 17,0 & 11,9 & 4,0 & 29,2 \\
\hline Mato Grosso do Sul & 0,6 & 13,3 & 11,4 & 7,2 & 4,2 & 17,0 \\
\hline Mato Grosso & 1,3 & 13,3 & 14,4 & 11,5 & 2,6 & 30,4 \\
\hline Goiás & 4,2 & 28,8 & 21,2 & 15,6 & 5,0 & 31,6 \\
\hline Distrito Federal & 1,1 & 13,9 & 15,6 & 7,4 & 3,0 & 36,8 \\
\hline BR & 4,0 & 22,7 & 21,0 & 10,7 & 8,2 & 36,7 \\
\hline
\end{tabular}

Fonte: Sistema de Informações Sobre Mortalidade (SIM) 
A variável naturalidade também teve boa completude para a maior parte dos estados, contudo a variação entre eles foi mais ampla, apresentando maior quantidade de estados com preenchimento regular (Espírito Santo, 16,2\%; Pará, 10\%; e Rondônia, 17,2\%) e até mesmo ruim (Rio de Janeiro, 29,8\%; Alagoas, 20,3\%).

Nenhuma das demais variáveis apresentou completude satisfatória, o que pode prejudicar as análises que as utilizem para verificar fatores associados à causa de morte.

Na Tabela 4, é possível observar os resultados de completude agregada por municípios. A avaliação da qualidade da informação municipal é importante, uma vez que a TMP deve ser calculada para todos os níveis de gestão. Por isso, foram analisadas as principais variáveis utilizadas para o estudo de desigualdade: raça/cor, estado civil e escolaridade. Foi verificado que aproximadamente $80 \%$ dos municípios apresentavam completude excelente quanto à variável raça/cor, 31,2\%, quanto ao estado civil, e apenas $8,2 \%$, quanto à escolaridade. Isso mostra que mesmo a variável de melhor preenchimento (raça/cor) ainda apresenta um número muito elevado de municípios onde a completude é regular, ruim ou muito ruim.

Tabela 4. Classificação dos municípios quanto à completude das variáveis raça/cor, escolaridade e estado civil. Brasil, 2016

\begin{tabular}{|c|c|c|c|c|c|}
\hline Variável & \multicolumn{2}{|c|}{ Classificação } & \multirow{2}{*}{$\begin{array}{c}n \\
4306\end{array}$} & \multirow{2}{*}{$\begin{array}{c}\text { \% válida } \\
78,3\end{array}$} & \multirow{2}{*}{$\begin{array}{c}\% \\
\text { acumulativa } \\
78,3\end{array}$} \\
\hline \multirow{6}{*}{ Raça/cor } & Excelente & $<5 \%$ & & & \\
\hline & Bom & $5 \%-<10 \%$ & 786 & 14,3 & 92,6 \\
\hline & Regular & $10 \%-<20 \%$ & 311 & 5,7 & 98,3 \\
\hline & Ruim & $20 \%-<50 \%$ & 87 & 1,6 & 99,9 \\
\hline & Muito ruim & $>=50 \%$ & 6 & 1 & 100,0 \\
\hline & & Total & 5496 & 100,0 & \\
\hline \multirow{6}{*}{ Escolaridade } & Excelente & $<5 \%$ & 441 & 8,2 & 8,2 \\
\hline & Bom & $5 \%-<10 \%$ & 559 & 10,4 & 18,6 \\
\hline & Regular & $10 \%-<20 \%$ & 1767 & 32,8 & 51,4 \\
\hline & Ruim & $20 \%-<50 \%$ & 2318 & 43,1 & 94,5 \\
\hline & Muito ruim & $>=50 \%$ & 294 & 5,5 & 100,0 \\
\hline & & Total & 5379 & 100,0 & \\
\hline \multirow{6}{*}{ Estado civil } & Excelente & $<5 \%$ & 1706 & 31,4 & 31,4 \\
\hline & Bom & $5 \%-<10 \%$ & 1711 & 31,5 & 62,8 \\
\hline & Regular & $10 \%-<20 \%$ & 1378 & 25,3 & 88,1 \\
\hline & Ruim & $20 \%-<50 \%$ & 547 & 10,1 & 98,2 \\
\hline & Muito ruim & $>=50 \%$ & 98 & 1,8 & 100,0 \\
\hline & & Total & 5440 & 100,0 & \\
\hline
\end{tabular}

Fonte: Sistema de Informações Sobre Mortalidade (SIM)

\section{DISCUSSÃO}

A qualidade dos insumos para o cálculo do indicador de TMP depende da clareza metodológica, acessibilidade, oportunidade dos dados e completude das variáveis. O SIM tem boa clareza metodológica, visto que seu instrumento de coleta, informações registradas, periodicidade e disponibilização se adequam aos parâmetros internacionais ${ }^{6}$. 
No que tange à acessibilidade dos dados, o SIM se mostra como uma poderosa fonte de informações para a gestão do Sistema Único de Saúde (SUS) diante de seu caráter contínuo, abrangência universal, regularidade, boa cobertura e acesso livre ${ }^{12}$, contando ainda com a disponibilidade do banco completo e desagregável pela internet, que é uma vantagem que poucos países da região dispõem ${ }^{6}$

A coleta de dados do SIM é de responsabilidade dos estados e municípios, por meio das suas respectivas secretarias de saúde, o que reforça a adequação do sistema aos princípios de descentralização, regionalização e municipalização da saúde no âmbito do SUS. As informações do SIM podem ser desagregadas, permitindo seu uso para os níveis de gestão federal, estadual e municipal. Por motivos de segurança e privacidade, não são disponibilizadas informações desagregadas a ponto de permitir a identificação dos indivíduos.

Sobre a oportunidade dos dados, a defasagem de cerca de dois anos entre a data de ocorrência do óbito e a disponibilidade das bases de dados limita o monitoramento e a vigilância dos eventos. Contudo, dados da OMS sugerem que a oportunidade dos dados de mortalidade no Brasil está de acordo com a de países considerados desenvolvidos e com a alta qualidade de sistemas de mortalidade, como Estados Unidos, Inglaterra e Canadá13.

A respeito da completude da causa básica de óbito, foi observado que mais de $20 \%$ dos municípios ainda apresentavam qualidade inadequada do preenchimento das causas de óbito. $\mathrm{Na}$ análise por indivíduo, não foi possível verificar tal achado com tanta clareza, pois as DO com causas mal definidas eram diluídas no volume de óbitos das regiões.

A diferença de completude da causa de óbito entre as faixas etárias foi superada, e, em 2016, algumas abrangências já apresentavam melhor completude para a faixa de 60 a 69 em relação aos mais jovens.

A completude das informações no Norte e no Nordeste se aproximou do nível nacional, mas ainda estava mais elevada que as regiões Sul e Centro-Oeste. Uma possível explicação para tal fenômeno seria o aumento na estrutura assistencial nesses locais ${ }^{14}$.

O preenchimento da causa básica de óbito reflete também a disponibilidade de infraestrutura assistencial e de condições para o diagnóstico de doenças e a capacitação profissional para preenchimento da $\mathrm{DO}^{14}$. Quando determinado local apresenta elevado grau de mau preenchimento da causa de óbito, torna-se difícil estimar indicadores confiáveis a partir dessa informação.

Achados de estudos anteriores já indicavam que a qualidade do preenchimento da causa básica de óbito no SIM é considerada deficiente quando relacionada à mortalidade infantil ${ }^{15}$. Contudo, este é o primeiro estudo que mostra que a situação é similar para a mortalidade prematura por DCNT em diversas abrangências geográficas.

Os dados coletados mostram que ainda há muitos municípios que não apresentavam qualidade de informação epidemiológica adequada para o cálculo de indicadores. É importante ressaltar a relevância de conhecer a qualidade da informação do óbito antes de calcular a TMP, visto que, com o elevado número de informações em branco, o indicador pode ser super ou subestimado.

Sobre a completude das variáveis sociodemográficas, por sua vez, é possível afirmar que é necessário realizar esforços para melhorar a qualidade do preenchimento de todas as variáveis. Foi verificado um número elevado de municípios onde a completude dessas variáveis era regular, ruim ou muito ruim. Assim, nesses municípios, é preciso questionar a confiabilidade das aferições realizadas relacionando as condições de saúde com a TMP.

O esforço deve ser realizado, principalmente, na variável escolaridade. A escolaridade é apontada no Plano de Ações Estratégicas para o Enfrentamento das Doenças Crônicas Não Transmissíveis como um ponto a ser a estudado para o combate dessas doenças. Esse fator é importante, porque, embora as DCNT atinjam indivíduos de todas as camadas socioeconômicas, elas afetam especialmente os grupos mais vulneráveis, como os idosos e as pessoas de baixa escolaridade e renda ${ }^{16}$. Apesar disso, as disparidades na incompletude dessa variável ainda são notáveis. Por esse motivo, melhorar a qualidade de seu preenchimento por meio da sensibilização dos profissionais da área da saúde quanto ao seu potencial se mostra uma medida necessária. 
A raça/cor, apesar de menos incompleta, ainda mostrou resultados insatisfatórios para algumas abrangências. A completude dessa variável teve muitos avanços, e isso é importante porque esse tipo de informação é de relevância conhecida para a avaliação das políticas de saúde. Isso ocorre porque, em razão do legado histórico de discriminação e exploração, as cores da pele negra ou parda passaram a constituir um indicador sensível de desigualdade nas condições de vida, saúde e morte ${ }^{17,18,19}$. Portanto, esse tipo de informação é essencial para traçar o perfil de mortalidade da população e, com isso, contribuir para a equidade na prestação de serviços de saúde. Esse fato é frequentemente reconhecido pelo Estado brasileiro, como é possível observar, por exemplo, na Política Nacional de Informação e Informática em Saúde ${ }^{20} \mathrm{e}$ na Política Nacional de Saúde Integral da População Negra ${ }^{21}$.

É interessante notar que, apesar de o SIM possuir variáveis socioeconômicas, poucos trabalhos o utilizaram como fonte de informação para a identificação de desigualdades na mortalidade. Após uma revisão bibliográfica, dos 34 estudos encontrados sobre o tema na Biblioteca Virtual em Saúde, apenas 8 utilizaram informações socioeconômicas e demográficas desse sistema22.

Em geral, os estudos encontrados relacionaram a mortalidade com as condições socioeconômicas obtidas a partir de censos (IBGE), inquéritos e pesquisas, ainda que sua abrangência seja inferior à do SIM. Isso pode demonstrar certa desconfiança da comunidade acadêmica quanto à qualidade da informação socioeconômica do SIM.

Os problemas do uso de fontes de informação como inquéritos e pesquisas são as limitações no que se refere à periodicidade e ao nível de desagregação dos dados ${ }^{23}$, principalmente em razão da expansão do papel dos municípios na formulação, acompanhamento e implementação de programas de saúde, que vêm aumentando com os processos de descentralização e regionalização do SUS, iniciados com a Norma Operacional de Assistência à Saúde do SUS (NOAS-SUS) 01/2001 e aprimorados pelo Pacto pela Saúde, de 2006, e, depois, pelo Contrato Organizativo de Ação Pública (COAP), de $2011^{24}$.

É importante notar que, embora o SIM apresente uma série de vantagens, uma de suas maiores limitações é não captar informações sobre fatores de risco, como fatores comportamentais e de estilo de vida. Contudo, de um modo geral, os achados deste artigo apontam que as variáveis escolaridade, raça/cor e estado civil podem e devem ser utilizadas para verificar a relação entre a TMP e a desigualdade social na saúde, e o SIM se mostra uma importante fonte desses dados. É recomendado que, antes de estimar a taxa, sejam levados em consideração os aspectos da qualidade da informação aqui mencionados.

\section{CONSIDERAÇÕES FINAIS}

O acompanhamento das mortes prematuras por DCNT na população brasileira é certamente um assunto muito importante para a saúde pública, e sua importância é crescente em razão de sua participação cada vez maior nas causas de óbito da população. A TMP é um indicador muito relevante para conhecer a situação de saúde de determinado local ou acompanhar os programas e políticas propostos nacionalmente para todas as abrangências geográficas.

Quanto à qualidade da informação, o SIM se mostrou uma fonte adequada de dados para estimar a TMP. Suas variáveis, periodicidade e cobertura estão de acordo com os parâmetros internacionais. Além disso, o sistema disponibiliza todas as informações gratuitamente para acesso livre e universal por meio da plataforma on-line do DATASUS.

Apesar disso, a qualidade da informação da causa básica de óbito apresenta grande variação em termos de abrangência geográfica. Embora a maioria dos municípios brasileiros tenha boa completude da informação da causa básica de óbitos, muitos ainda possuem péssimo preenchimento, sendo necessário identificá-los para não estimar um indicador pouco confiável. Um dos possíveis motivos para o mau preenchimento das causas de óbito pode ser a falta de recursos de diagnóstico em algumas localidades, tanto físicos quanto humanos. A rede de atenção, os serviços e a tecnologia disponíveis são fatores-chave para a identificação correta das causas de óbito. 
O mesmo ocorre quanto à qualidade das informações das variáveis socioeconômicas. Para analisar as desigualdades sociais relacionadas à mortalidade prematura por DCNT, é preciso verificar a completude dessas informações para a abrangência selecionada. Vale ressaltar que a perda de informações pode ser mais acentuada em populações mais vulneráveis, o que prejudica a identificação e o enfrentamento das desigualdades na mortalidade.

\section{REFERÊNCIAS}

1. Campolina AG, Adami F, Santos JL, Lebrão ML. A transição de saúde e as mudanças na expectativa de vida saudável da população idosa: possíveis impactos da prevenção de doenças crônicas. Cad Saude Publica. 2013;29(6):1217-29. http://dx.doi.org/10.1590/S0102-311X2013000600018. PMid:23778553.

2. WHO. Health in 2015: from MDGs, Millennium Development Goals to SDGs, Sustainable Development Goals. Geneve: WHO; 2015.

3. Brasil. Ministério da Saúde, Secretaria de Vigilância em Saúde, Departamento de Análise de Situação de Saúde. Plano de ações estratégicas para o enfrentamento das doenças crônicas não transmissíveis (DCNT) no Brasil 2011-2022. Brasília: Editora do Ministério da Saúde; 2011.

4. Jannuzzi PD. Indicadores para diagnóstico, monitoramento e avaliação de programas sociais no Brasil. Revista do Serviço Público. 2005;56(2):137-60. http://dx.doi.org/10.21874/rsp.v56i2.222.

5. Brasil. Ministério da Saúde. A experiência brasileira em sistemas de informação em saúde. Brasília: Editora do Ministério da Saúde; 2009.

6. Romero D, Cunha CB. Avaliação da qualidade das variáveis sócio-econômicas e demográficas dos óbitos de crianças menores de um ano registrados no Sistema de Informações sobre Mortalidade do Brasil (1996/2001). Cad Saude Publica. 2006;22(3):673-84. http://dx.doi.org/10.1590/S0102-311X2006000300022. PMid:16583111.

7. Organização Mundial da Saúde. Universidade de São Paulo. CID-10 Classificação Estatística Internacional de Doenças e Problemas Relacionados à Saúde. São Paulo: EdUSP; 1997.

8. Kanso S, Romro DE, Leite IDC, Moraes END. Diferenciais geográficos, socioeconômicos e demográficos da qualidade da informação da causa básica de morte dos idosos no Brasil. Cad Saúde Pública. 2011;27(7):1323-39.

9. Romero DE, Cunha CB. Avaliação da qualidade das variáveis epidemiológicas e demográficas do Sistema de Informações sobre Nascidos Vivos, 2002. Cad Saude Publica. 2007;23(3):701-14. http://dx.doi.org/10.1590/ S0102-311X2007000300028. PMid:17334583.

10. Brasil. Ministério da Saúde. A declaração de óbito: documento necessário e importante. 3. ed. Brasília: Ministério da Saúde; 2009.

11. Kanso S, Romero D, Leite I, Marques A. A evitabilidade de óbitos entre idosos em São Paulo, Brasil: análise das principais causas de morte. Cad Saude Publica. 2013;29(4):735-48. http://dx.doi.org/10.1590/S0102311X2013000800011. PMid:23568303.

12. Lima EEC, Queiroz BL. Evolution of the deaths registry system in Brazil: associations with changes in the mortality profile, under-registration of death counts, and ill-defined causes of death. Cad Saude Publica. 2014;30(8):1721-30. http://dx.doi.org/10.1590/0102-311X00131113. PMid:25210911.

13. Mathers CD, Fat DM, Inoue M, Rao C, Lopez AD. Counting the dead and what they died from: an assessment of the global status of cause of death data. Bull. World Health Organ. 2005;83(3):171-177.

14. Vanderlei LC, Arruda BK, Frias PG, Arruda S. Avaliação da confiabilidade da causa básica de óbito em unidade terciária de atenção à saúde materno-infantil. Inf Epidemiol Sus. 2002;11(1):15-23. http://dx.doi. org/10.5123/S0104-16732002000100003.

15. Barbeiro FM, Fonseca SC, Tauffer MG, Ferreira MS, Silva FP, Ventura PM, et al. Fetal deaths in Brazil: a systematic review. Rev Saude Publica. 2015;49:22. http://dx.doi.org/10.1590/S0034-8910.2015049005568. PMid:25902565.

16. Malta DC, Silva JB Jr. Plano de Ações Estratégicas para o Enfrentamento das Doenças Crônicas Não Transmissíveis no Brasil após três anos de implantação, 2011-2013. Epidemiol Serv Saude. 2014;23(3):38998. http://dx.doi.org/10.5123/S1679-49742014000300002.

17. Araújo EM, Costa M CN, Hogan VK, Araújo TM, Dias AB, Oliveira LOA. A utilização da variável raça/cor em Saúde Pública: possibilidades e limites. Interface. 2009;13(31):383-94.

18. Marmot M. Social determinants of health inequalities. Lancet. 2005;365(9464):1099-104. http://dx.doi org/10.1016/S0140-6736(05)71146-6. PMid:15781105. 
19. Kabad JF, Bastos JL, Santos RV. Raça, cor e etnia em estudos epidemiológicos sobre populações brasileiras: revisão sistemática na base PubMed. Physis Rev Saúde Coletiva. 2012;22(3):895-918. http://dx.doi. org/10.1590/S0103-73312012000300004.

20. Brasil. Ministério da Saúde, Secretaria-Executiva, Departamento de Monitoramento e Avaliação do SUS. Política Nacional de Informação e Informática em Saúde. Brasília: Editora Ministério da Saúde; 2016.

21. Brasil. Ministério da Saúde, Secretaria de Gestão Estratégica e Participativa, Departamento de Apoio à Gestão Participativa e ao Controle Social. Política Nacional de Saúde Integral da População Negra: uma política para o SUS. Brasília: Editora do Ministério da Saúde; 2007.

22. Rodrigues JM. Análise da qualidade da informação, potencialidades e limitações do indicador de mortalidade prematura por doenças crônicas não transmissíveis e sua utilização nos Objetivos do Desenvolvimento Sustentável [dissertação]. Rio de Janeiro: Fundação Oswaldo Cruz; 2017.

23. Viacava F, Dachs N, Travassos C. Os inquéritos domiciliares e o Sistema Nacional de Informações em Saúde. Cien Saude Colet. 2006;11(4):863-9. http://dx.doi.org/10.1590/S1413-81232006000400002.

24. Romero DE, Pires DC, Marques A, Muzy J. Diretrizes e indicadores de acompanhamento das políticas de proteção à saúde da pessoa idosa no Brasil. Reciis. 2019;13(1):134-57. http://dx.doi.org/10.29397/reciis. v13i1.1569.

25. Brasil. Ministério da Saúde. Banco de dados do Sistema Único de Saúde - DATASUS. Informações de Saúde, Sistema de Informações sobre Mortalidade [Internet]. Disponível em: https://datasus.saude.gov. $\mathrm{br} /$ transferencia-de-arquivos/. 